\title{
Enzymatic profiling of cellulosomal enzymes from the human gut bacterium, Ruminococcus champanellensis, reveals a fine-tuned system for cohesin-dockerin recognition
}

\author{
Sarah Moraïs, ${ }^{1}$ Yonit Ben David, ${ }^{1}$ Lizi Bensoussan, ${ }^{1}$ \\ Sylvia H. Duncan, ${ }^{2}$ Nicole M. Koropatkin, ${ }^{3}$ \\ Eric C. Martens, ${ }^{3}$ Harry J. Flint ${ }^{2}$ and \\ Edward A. Bayer ${ }^{1 *}$ \\ ${ }^{1}$ Department of Biological Chemistry, The Weizmann \\ Institute of Science, Rehovot, Israel. \\ ${ }^{2}$ Microbiology Group, Rowett Institute of Nutrition and \\ Health, University of Aberdeen, Aberdeen, UK. \\ ${ }^{3}$ Department of Microbiology and Immunology, \\ University of Michigan Medical School, Ann Arbor, MI \\ 48109, USA.
}

\section{Summary}

Ruminococcus champanellensis is considered a keystone species in the human gut that degrades microcrystalline cellulose efficiently and contains the genetic elements necessary for cellulosome production. The basic elements of its cellulosome architecture, mainly cohesin and dockerin modules from scaffoldins and enzyme-borne dockerins, have been characterized recently. In this study, we cloned, expressed and characterized all of the glycoside hydrolases that contain a dockerin module. Among the 25 enzymes, 10 cellulases, 4 xylanases, 3 mannanases, 2 xyloglucanases, 2 arabinofuranosidases, 2 arabinanases and one $\beta$-glucanase were assessed for their comparative enzymatic activity on their respective substrates. The dockerin specificities of the enzymes were examined by ELISA, and 80 positives out of 525 possible interactions were detected. Our analysis reveals a finetuned system for cohesin-dockerin specificity and the importance of diversity among the cohesin-dockerin sequences. Our results imply that cohesin-dockerin pairs are not necessarily assembled at random among the same specificity types, as generally believed for other cellulosome-producing bacteria, but reveal a

Received 16 July, 2015; revised 2 September, 2015; accepted 2 September, 2015. $\quad{ }^{*}$ For correspondence. E-mail ed.bayer@ weizmann.ac.il; Tel. (+972) 8934 2373; Fax (+972) 89344118. more organized cellulosome architecture. Moreover, our results highlight the importance of the cellulosome paradigm for cellulose and hemicellulose degradation by $R$. champanellensis in the human gut.

\section{Introduction}

Interest in the human gut microbiota has increased considerably during recent years due to its influence upon human health. One of the main activities of the gut microbiota is to ferment fibre derived from the diet that remains undigested by host enzymes, yielding additional metabolites and energy sources that influence host metabolism, e.g. nutrient absorption and production (Goodman et al., 2009) and energy balance (Turnbaugh et al., 2006). In addition, the human gut microbiome plays a role in the regulation of the immune system (Lee and Mazmanian, 2010) and is an important parameter in many inflammatory and infectious diseases (Young et al., 2005; Kerckhoffs et al., 2011; Vaarala, 2012).

Although cellulose is the major constituent of plant fibre, there have been very few reports of bacteria from the human gut that are able to degrade cellulose. To date, the only human colonic bacterium reported to be capable of degrading crystalline cellulose is Ruminococcus champanellensis. This anaerobic, cellulolytic, Grampositive bacterium has been isolated from the human colon and characterized (Chassard et al., 2012). An additional strain closely related to $R$. champanellensis, Ruminococcus sp. CAG:379, was isolated independently from the human gut microbiota, suggesting that this bacterium could be widespread in humans. In view of $R$. champanellensis remarkably efficient enzymatic activity on microcrystalline cellulose, its genome was sequenced (GenBank, FP929052.1), and this revealed numerous genes coding for elements of a cellulosomal enzyme complex (Ben David et al., 2015), including 12 scaffoldin proteins, collectively carrying 20 cohesins and 65 dockerin-containing proteins.

Cellulosomes are high-molecular-weight multi-enzymes complexes that were first described in the anaerobic highly cellulolytic thermophilic bacterium, Clostridium 
thermocellum (Lamed et al., 1983). One of its basic cellulosomal components is a cell-associated scaffoldin subunit, which contains a single cellulose-binding module (CBM) for substrate binding and nine cohesin modules that serve to integrate dockerin-containing enzymes. The high-affinity cohesin-dockerin interaction was demonstrated to be calcium-dependent (Yaron et al., 1995) and species-specific (Lytle et al., 1996). To date, three types of cohesins or dockerins have been defined, based on their amino acid sequences (Bayer et al., 2004). The proximity of the enzymes within the complex, the targeting of the complex to the substrate and its anchoring to the cell surface are believed to render the cellulosomal complex highly efficient in cellulose degradation. Cellulosomes with various architectures were also discovered in additional anaerobic bacteria within the Clostridiaceae and also within the Ruminococcaceae, specifically Ruminococcus flavefaciens isolated from the cow rumen (Ding et al., 2001; Rincon et al., 2010). The latter possesses a larger variety of cellulosomal components, including a large set of adaptor scaffoldins, but its cellulosomal organization has yet to be fully determined.

In a recent study (Ben David et al., 2015), the in vitro characterization of the various cellulosomal components of R. champanellensis was performed. The cohesindockerin interactions among the components revealed the possible assembly of a cell-associated cellulosomal complex that could assemble up to 11 enzymes. In addition, a scaffoldin cluster was described, displaying organizational similarities with the $R$. flavefaciens scaffoldin cluster. Moreover, most of the cohesins of the two species appeared to be phylogenetically related (in most cases type III cohesins). The reiterated sequences of the 65 dockerins were divided into four groups, using bioinformatic-based criteria. Twenty-four selected representatives of each group were examined for their specificities, among them 8 originating from scaffoldins and 11 derived from putative glycoside hydrolases. The enzymatic activity of each protein, however, remained undefined.

In the present report, we aimed to characterize the enzymatic activity of the 25 dockerin-containing glycoside hydrolases revealed by CAZy and bioinformatic analysis, along with their dockerin specificities, in order to expand our knowledge on the architecture and activity of the cellulosome from $R$. champanellensis, thus far the sole characterized cellulosome-producing bacterium in the human gut.

\section{Results}

\section{Dockerin-containing glycoside hydrolase production}

Bioinformatic analysis of the $R$. champanellensis genome revealed 25 putative dockerin-containing glycoside hydrolases (Ben David et al., 2015). Accordingly, these putative enzymes appeared to be related to $\mathrm{GH} 5, \mathrm{GH} 8$, GH9, GH10, GH11, GH16, GH26, GH30, GH43, GH44, GH48, GH74 and GH98 families (Cantarel et al., 2009). The modular organization and molecular weights of these GHs are listed in Table 1. Altogether, 25 putative enzymes were cloned without their signal peptides, taking into account their inherent modular organization. The cloning of two multifunctional proteins, GH9B-Doc-GH16A and GH43C-Doc-CE, presented technical difficulties [no amplification could be obtained using either genomic DNA or whole cells as template, under various polymerase chain reaction (PCR) conditions]; therefore, they were cloned in segments. In the first, GH9B-Doc-GH16A, the dockerin and the GH16 module only could be inserted in the plasmid, and in the second GH43C-Doc-CE, the carbohydrate esterase module (CE), was omitted in the final construct. In both cases, the dockerin module, which may bear the most valuable information for our studies, could be preserved.

All of the proteins were produced in Escherichia coli, and SDS-PAGE analysis of the purified proteins revealed in most cases a major protein band in good agreement with the respective calculated molecular masses (Fig. S1).

\section{Enzymatic activity profile of the GH modules}

The enzymes GH5A, GH5B, GH8A, GH9A, GH9C, GH9D, GH9E, GH9F and GH9G were all active on carboxymethyl cellulose (CMC) and Avicel (microcrystalline cellulose), and thus classified as endoglucanases (Fig. 1A and B). Only endoglucanases or processive endoglucanases are active on the soluble CMC substrate, whereas exoglucanase activity can be detected on microcrystalline cellulose. Three of the enzymes, GH8A, GH9A and GH9D, were the most active on the CMC substrate, and GH9A and GH9D also exhibited the highest levels of degradation on Avicel. As expected, the GH48A enzyme exhibited very low levels of enzymatic activity on Avicel by itself, but acted in synergy with GH5B and GH8A (2.7- and 4-fold, respectively), in accordance with other common cellulosomal GH48 cellobiohydrolases (Vazana et al., 2010; Zhang et al., 2010; Morais et al., 2012). Analysis of cellassociated proteins revealed that the two proteins most highly upregulated during growth of $R$. champanellensis on filter paper cellulose compared with growth on cellobiose were Cel48A (GH48A, 364-fold increase) and Cel9F (GH9F 186-fold increase) enzymes (Table 2, Fig. S2). In addition, a number of gene products showed decreased expression during growth on cellulose, which may be related to slower growth rate.

The GH10A, GH10B-GH43E, GH11A-CE and GH30A-CE were active on beechwood xylan and thus classified as xylanases (Fig. 2A). The GH10A and 


\section{S. Moraïs et al.}

Table 1. Putative dockerin-containing glycoside hydrolases of $R$. champanellensis.

\begin{tabular}{|c|c|c|c|c|}
\hline GH family & Current nomenclature & Modular organization & Molecular weight ${ }^{a}$ & Gl number \\
\hline \multirow[t]{3}{*}{ GH5 } & GH5A & GH5A-Doc & $68695 \mathrm{Da}$ & 291543414 \\
\hline & GH5B & GH5B-Doc & $63394 \mathrm{Da}$ & 291543738 \\
\hline & GH5C & GH5C-Doc & $68852 \mathrm{Da}$ & 291545071 \\
\hline GH8 & GH8A & GH8A-Doc & $51881 \mathrm{Da}$ & 291543899 \\
\hline \multirow[t]{7}{*}{ GH9 } & GH9A & GH9A-CBM3c'-Doc & $93340 \mathrm{Da}$ & 291543282 \\
\hline & GH9B & $\begin{array}{l}\text { CBM4-Fn3-GH9B-Doc-GH16A } \\
(\text { Doc-GH16A) }\end{array}$ & $\begin{array}{l}114666 \mathrm{Da} \\
36367 \mathrm{Da}\end{array}$ & 291543673 \\
\hline & GH9C & GH9C-CBM3c'-Doc & $98050 \mathrm{Da}$ & 291543938 \\
\hline & GH9D & GH9D-Doc & $64377 \mathrm{Da}$ & 291544445 \\
\hline & GH9E & GH9E-CBM3c-Doc & $82111 \mathrm{Da}$ & 291544574 \\
\hline & GH9F & CBM4-Fn3-GH9F-Doc & $104833 \mathrm{Da}$ & 291544575 \\
\hline & GH9G & GH9G-CBM3c-Doc & $79955 \mathrm{Da}$ & 291545280 \\
\hline \multirow[t]{2}{*}{$\mathrm{GH} 10$} & $\mathrm{GH} 10 \mathrm{~A}$ & CBM4-GH10A-Doc & $69424 \mathrm{Da}$ & 291543470 \\
\hline & GH10B-GH43E & CBM4-GH10B-CBM4-Doc-GH43E-CBM6 & $137621 \mathrm{Da}$ & 291544573 \\
\hline GH11 & GH11A-CE & GH11A-CBM4-Doc-CBM4-CE & $94529 \mathrm{Da}$ & 291545196 \\
\hline \multirow[t]{2}{*}{ GH26 } & GH26A & CBM6-GH26A-CBM6-Doc & $79544 \mathrm{Da}$ & 291544512 \\
\hline & $\mathrm{GH} 26 \mathrm{~B}$ & CBM6-GH26B-Doc & $68166 \mathrm{Da}$ & 291545037 \\
\hline $\mathrm{GH} 30$ & $\mathrm{GH} 30 \mathrm{~A}$ & GH30A-CBM4-Doc-CE & $104949 \mathrm{Da}$ & 291544794 \\
\hline \multirow[t]{5}{*}{$\mathrm{GH} 43$} & $\mathrm{GH} 43 \mathrm{~A}$ & GH43A-CBM61-X157-Doc & $79531 \mathrm{Da}$ & 291543994 \\
\hline & GH43B & GH43B-CBM6-Doc & $80395 \mathrm{Da}$ & 291543991 \\
\hline & $\mathrm{GH} 43 \mathrm{C}$ & GH43C-CBM4-Doc-CE & $118020 \mathrm{Da}$ & 291544122 \\
\hline & & GH43C-CBM4-Doc ${ }^{\mathrm{C}}$ & $83891 \mathrm{Da}$ & \\
\hline & GH43D & GH43D-Doc & $83137 \mathrm{Da}$ & 291544405 \\
\hline GH44 & GH44A & GH44A-Doc & $81929 \mathrm{Da}$ & 291543699 \\
\hline $\mathrm{GH} 48$ & $\mathrm{GH} 48 \mathrm{~A}$ & GH48A-Doc & $88132 \mathrm{Da}$ & 291544207 \\
\hline GH74 & GH74A & GH74A-Doc & $92496 \mathrm{Da}$ & 291543413 \\
\hline GH98 & GH98A & GH98A-CBM35-X157-Doc & $114519 \mathrm{Da}$ & 291544973 \\
\hline
\end{tabular}

Abbreviations used in the table: GH, glycoside hydrolase; Doc, dockerin; CBM, carbohydrate binding module; Fn3, fibronectin type 3 motif; CE, carbohydrate esterase; X157, domain of unknown function.a. Based on the known amino acid composition of the desired protein using the PROTPARAM tool (http://www.expasy.org/tools/protparam.html).b. Entire enzyme could not be cloned, only the GH16 and dockerin modules.c. The complete protein could not be cloned, and the CE module was thus omitted.

GH11A-CE exhibited the highest level of degradation, while GH30A-CE was the least active of these four xylanases. Three mannanases, GH5C, GH26A and GH26B, were active on locust bean gum, and they exhibited similar levels of activity (Fig. 2B). GH16A was active on $\beta$-D-glucan from barley (Fig. 2C). Two arabinanases, GH43A and GH43D, were active on debranched arabinan, and GH43D was more active on this substrate (Fig. 2D). Two xyloglucanases, GH44A and GH74A, were active on xyloglucan, GH44A being more active (Fig. 2E). These two xyloglucanases were also active on CMC (but not PASC, phosphoric acid swollen cellulose) as sometimes observed for 'non-xyloglucanspecific xyloglucanases' (Zverlov et al., 2005). Two arabinofuranosidases, GH43B and GH10B-GH43E, were active on $\mathrm{pNP}-\alpha$-L-arabinofuranoside; the highest activity was measured for the bifunctional xylanasearabinofuranosidase GH10B-GH43E (Fig. 2A and F). Since arabinofuranosidase activity can be attributed only to the GH43 module, we presume that the xylanase activity of this bifunctional enzyme is provided by the $\mathrm{GH} 10$ module.

No enzymatic activity could be detected for $\mathrm{GH} 43 \mathrm{C}$ on xylans, arabinans, pNP- $\beta$-D-xylopyranoside and $p N P-\alpha-$ $\mathrm{L}$-arabinofuranoside. The fact that we had to truncate the enzyme for cloning considerations could be a reason for the apparent absence of enzymatic activity. In addition, the enzymatic activity of GH98A remains undetermined (no enzymatic activity on pNP- $\beta$-D-galactopyranoside).

The schematic modular architecture of these 25 dockerin-containing glycoside hydrolases, along with their enzymatic activities and dockerin groupings and their proposed nomenclature, is presented in Fig. 3. In total, the enzymatic activities of 10 cellulases, 4 xylanases, 3 mannanases, 2 xyloglucanases, 2 arabinofuranosidases, 2 arabinanases and $1 \beta$-glucanase were established. The enzymatic activity of the putative carbohydrate-esterase modules remained undetermined.

\section{New insight into dockerin specificity: regrouping of dockerin-containing proteins}

Dockerin structures are characterized by two segments, each of which contains a $\mathrm{Ca}^{+2}$-binding loop and a cohesinbinding helix, which is coordinated by specific positions of amino acids (Pages et al., 1997; Mechaly et al., 2000; 2001; Carvalho et al., 2003; Bayer et al., 2004). Historically, in most of the type I dockerins, a clear twofold symmetry has been observed between their two segments, wherein designated recognition residues are 


\section{A CMC}

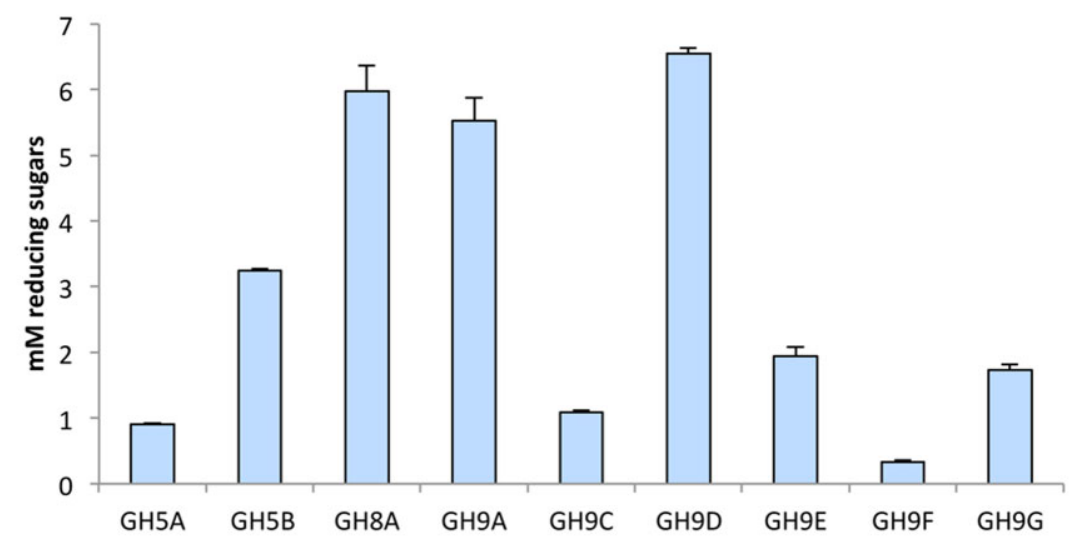

Fig. 1. Enzymatic activity of

R. champanellensis cellulosomal cellulases.

(A) Comparative enzymatic activity of the cellulases at a concentration of $0.5 \mu \mathrm{M}$.

Cellulases were tested at $\mathrm{pH} 5$ and $37^{\circ} \mathrm{C}$ for $1 \mathrm{~h}$ with $2 \%$ carboxymethyl cellulose. (B) Comparative enzymatic activity of cellulases at a concentration of $0.5 \mu \mathrm{M}$ and synergism with GH48A. Cellulases were tested at $\mathrm{pH} 5$ and $37^{\circ} \mathrm{C}$ for $24 \mathrm{~h}$ with $10 \%$ Avicel. Reactions were performed at least twice in triplicate; standard deviations are indicated.

\section{B Avicel}

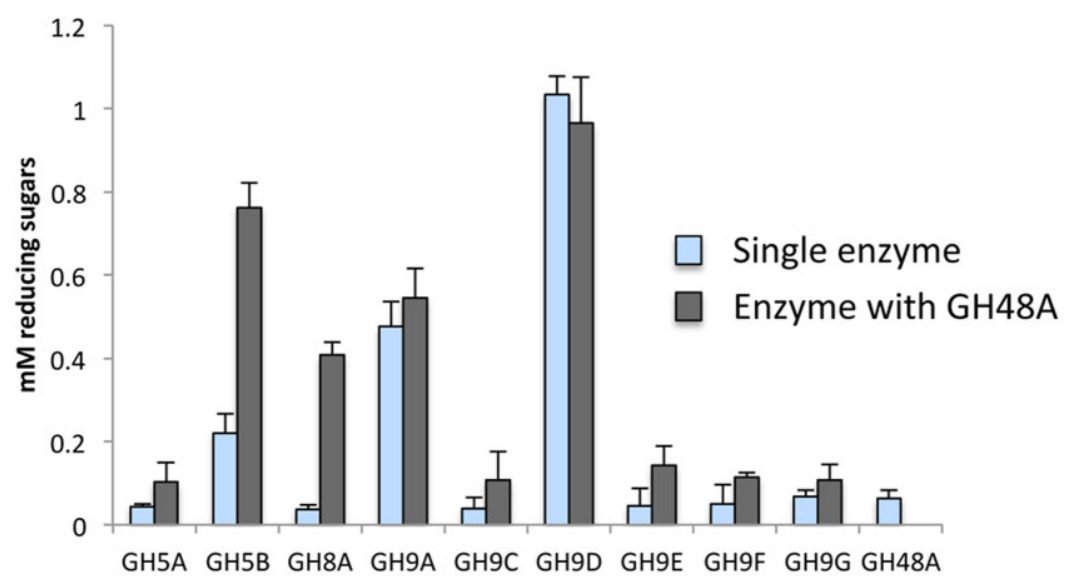

repeated (in identical or very similar fashion). This symmetry has proved to enable two separate cohesin-binding surfaces, with $180^{\circ}$ rotation between them, and this phenomenon has been termed the dual binding mode (Carvalho et al., 2007). However, it has also been observed that certain dockerins that lack structural symmetry display a single-binding mode (Bras et al., 2012). In those dockerin sequences, each segment represents separate binding interfaces that can recognize a different cohesin (Pinheiro et al., 2009; Voronov-Goldman et al., 2015). The latter characteristics of known dockerin sequences reflect the complexity and diversity in cohesin-dockerin interactions that contribute to dockerin flexibility in case of steric interferences and improved response to the dynamic process of plant cell wall degradation (Carvalho et al., 2007).

In our previous study (Ben David et al., 2015), the 64 dockerins of $R$. champanellensis (not including the ScaL dockerin) were aligned, and then clustered into four groups. Dockerins of Group 1 were found to interact directly with the cell-anchoring scaffoldin, ScaE. Most of the proteins in Group 2 represent glycoside hydrolase enzymes, mainly cellulases or closely associated enzymes. In contrast, in the dockerin-containing enzymes of Groups 3 and 4, most appeared to be hemicellulases as well as dockerin-containing proteins that lack confirmed carbohydrate-degrading components.

Intriguingly, the dockerins from Groups 3 and 4 exhibited similar binding profiles. Clearly, the dockerins of these two groups are asymmetrical in their 'repeated' segments, where the reputed recognition residues are clearly different in character (Ben David et al., 2015), thus indicating a single mode rather than a dual mode of binding. If so, then the two segments can theoretically be switched with retention of the same specificity characteristics. Therefore, a renewed alignment was performed, taking this possibility into account. Indeed, re-examination of the two dockerin segments in the sequences from Groups 3 and 4 revealed remarkable, but reversed, similarities between the two groups. Thus, the first binding interface of Group 
Table 2. Cell-associated proteins from R. champanellensis 18P13 cultures showing differential expression during growth on cellobiose or filter paper cellulose as sole energy sources.

\begin{tabular}{|c|c|c|c|c|c|c|}
\hline Spot ID & Fold-change & $P$-value & Protein hit & Score & $\%$ Coverage & Closest match to $R$. champanellensis $18 \mathrm{P} 13$ \\
\hline \multicolumn{7}{|c|}{ Filter paper cellulose $>$ cellobiose } \\
\hline 3620 & 364.2 & $<0.001$ & gi:291544207 & 960 & 23 & Cel48A \\
\hline 3606 & 185.6 & $<0.001$ & gi:291544575 & 1262 & 30 & Cel9F \\
\hline 4607 & 97.35 & $<0.001$ & gi:291543571 & 84 & 2 & Pyruvate, phosphate dikinase \\
\hline 2406 & 70.19 & $<0.001$ & gi:291544494 & 573 & 34 & Cell division protein FtsZ \\
\hline 7310 & 61.00 & 0.002 & gi:291544534 & 327 & 21 & Deoxy-D-arabinoheptulosonate phosphate synthase \\
\hline 6301 & 36.56 & 0.006 & gi:291544494 & 93 & 8 & Cell division protein FtsZ \\
\hline 7312 & 28.53 & 0.003 & gi:291543397 & 603 & 31 & Carbohydrate $\mathrm{ABC}$ transporter ATP BP CUT1 family \\
\hline 4108 & 12.32 & $<0.001$ & gi:291543600 & 308 & 37 & Hypothetical protein RUM04790 \\
\hline 4609 & 8.73 & 0.011 & gi:291544244 & 2207 & 9 & Elongation factor \\
\hline 7311 & 5.82 & $<0.001$ & gi:291545194 & 670 & 33 & Glutamate dehydrogenase \\
\hline 6307 & 2.10 & 0.002 & gi:291543339 & 175 & 12 & Branched chain amino acid transferase apoenzyme \\
\hline \multicolumn{7}{|c|}{ Cellobiose > filter paper cellulose } \\
\hline 4212 & 117.50 & 0.002 & gi:291543615 & 187 & 21 & CheY receiver domain \\
\hline 4403 & 81.50 & 0.039 & gi:524639232 & 716 & 39 & Elongation factor $\mathrm{Tu}$ \\
\hline 4206 & 77.17 & 0.026 & gi:291543975 & 343 & 42 & Tryptophan synthase \\
\hline 4404 & 67.63 & 0.031 & gi:291545113 & 712 & 18 & Hydroxy-methyl but-2-enyl phosphate reductase \\
\hline 6105 & 42.65 & 0.002 & gi:291544482 & 170 & 17 & SSU ribosomal protein S13P \\
\hline 4103 & 40.00 & 0.001 & gi:291544325 & 238 & 30 & Translation elongation factor EFP \\
\hline 5005 & 35.63 & 0.003 & gi:291544576 & 173 & 32 & Hypothetical protein RUM15970 \\
\hline 6204 & 11.86 & 0.009 & gi:291544387 & 251 & 28 & Transcription elongation factor GreA \\
\hline 6408 & 7.18 & 0.010 & gi:291543396 & 883 & 51 & Pyridoxal phosphate-dependent Trp B-like protein \\
\hline 5101 & 5.36 & 0.014 & gi:291544471 & 201 & 27 & SSU ribosomal protein S8P \\
\hline 3203 & 4.21 & 0.009 & gi:291544054 & 1041 & 63 & Fructose 1,6 bisphosphate aldolase \\
\hline 6107 & 3.14 & 0.005 & gi:291544476 & 232 & 22 & LSU ribosomal protein L15P \\
\hline 5004 & 3.03 & 0.010 & gi:291544048 & 146 & 22 & Uncharacterized protein \\
\hline
\end{tabular}

3 dockerins is highly similar to the second binding interface of Group 4 dockerins and vice versa (Fig. 4). According to this new arrangement of the dockerins, a mechanism of an alternative-binding mode can be suggested; it seems that the first segment of Group 3 and the second segment of Group 4 could be responsible for the binding to cohesins $C$ and $D$, while the second segment of Group 3 and the first segment of Group 4 would allow interaction with cohesin I. This hypothesis can also be extended to the Group 2 dockerins, which also bind to Cohl. Similar motifs were thus found between the first segment of the Group 2 dockerins and the segment that is considered to interact with Cohl in Groups 3 and 4, as described below (see Fig. 4). This analysis might therefore serve to explain why these groups are also associated with Cohl.

The Cohl-interacting dockerin segment is characterized by Val or lle and Ala (or small uncharged residue, i.e. Asn or Ser) in positions 10 and 11, mainly Val or an aliphatic residue in positions 13 and 14 (positions 14 and 15 in the second helix), and hydrophilic resides followed by aromatic amino acids in positions 17 and 19 (Fig. 4). In contrast, the sequences of the CohC- and CohDinteracting dockerin segment exhibit more variance in the amino acids found in the putative recognition positions. Yet the basic amino acids, Arg and Lys, exclusively occupy positions 17 and 18. Notably, the dockerins of Xyn11A-CE and GH98 have hydrophilic and charged amino acids in positions 10, 11 and 14 (position 15 in the second helix), which can explain why they failed to interact with Cohl.

According to these new findings, Groups 3 and 4 were redistributed into four groups of putative cohesin-dockerin interactions: (i) interaction with cohesins $\mathrm{C}, \mathrm{D}$ and I; (ii) interaction with CohC and CohD only; (iii) interaction with Cohl; and (iv) currently unknown interactions (Fig. S3).

\section{Affinity-based ELISA}

The dockerin-containing enzymes were examined for their interactions with the 21 cohesins of $R$. champanellensis. In a previous work (Ben David et al., 2015), 20 cohesins were described. The published sequence of the genome contains numerous gaps, and an additional scaffoldin, ScaL, was identified during the course of the present work. The ScaL gene, which was recovered by genome walking, includes a large $\mathrm{N}$-terminal domain of unknown function, a nucleoporin-like module, a cohesin module and a dockerin module (Table 3).

The cohesin genes were all fused with a CBM cassette that has been employed earlier for antigen recognition (Ben David et al., 2015). All 25 dockerin-bearing proteins were tested for their binding affinity with the 21 cohesins known to date, including the additional CohL from ScaL, described in this work. 


\section{A Beechwood xylan}

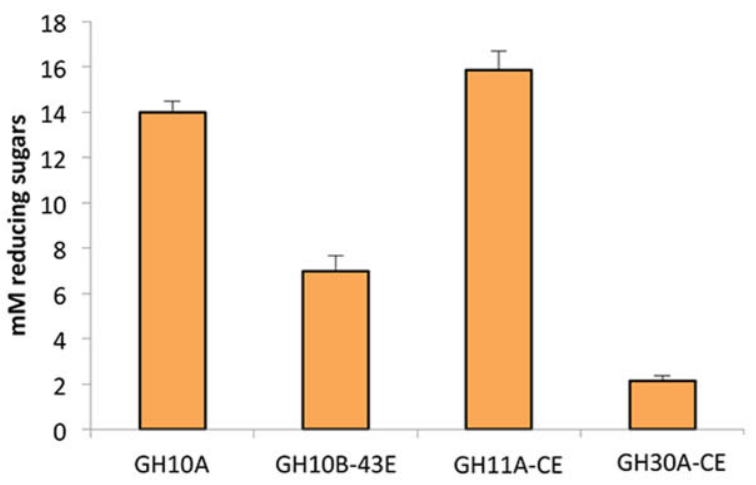

B Mannan

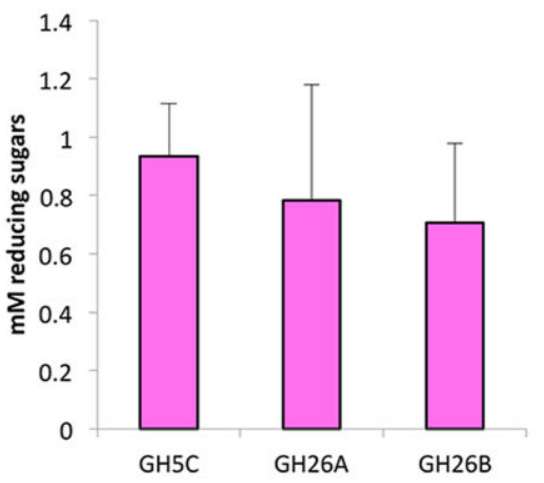

C Glucan

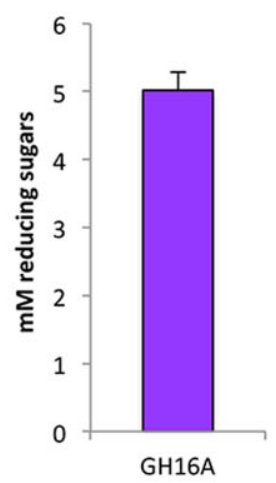

\section{Arabinan}

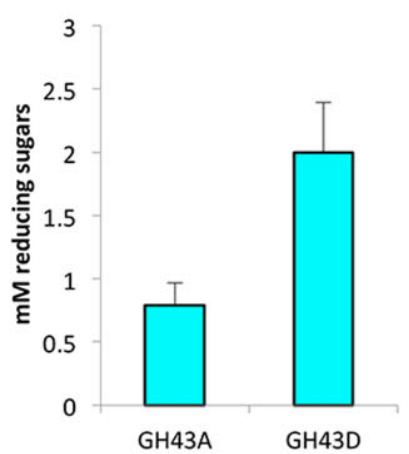

E Xyloglucan

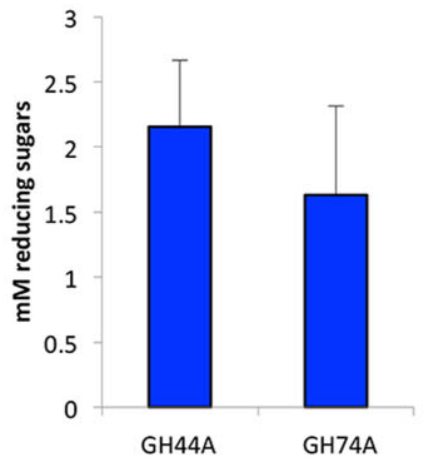

F pNP- $\alpha$-L-Arabinofuranoside

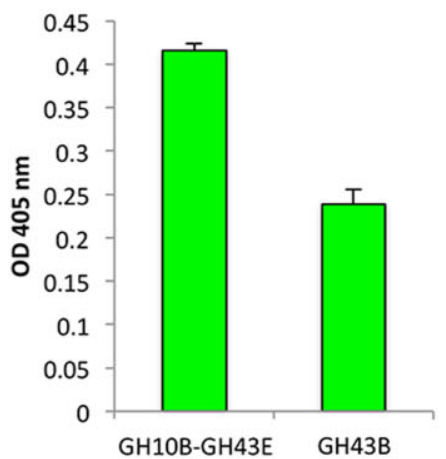

Fig. 2. Enzymatic profile of $R$. champanellensis cellulosomal glycoside hydrolases. (A) Comparative enzymatic activity of xylanases at a concentration of $0.5 \mu \mathrm{M}$ at $\mathrm{pH} 6$ and $37^{\circ} \mathrm{C}$ for $1 \mathrm{~h}$ with $2 \%$ beechwood xylan. (B) Comparative enzymatic activity of mannanases at a concentration of $0.5 \mu \mathrm{M}$ at $\mathrm{pH} 5$ and $37^{\circ} \mathrm{C}$ for $1 \mathrm{~h}$ with $1 \%$ locus bean gum. (C) $\beta$-glucanase activity at a concentration of $0.5 \mu \mathrm{M}$ at $\mathrm{pH} 5$ and $37^{\circ} \mathrm{C}$ for $1 \mathrm{~h}$ with $\beta$-D glucan from barley. (D) Comparative enzymatic activity of arabinanases at a concentration of $0.5 \mu \mathrm{M}$ at $\mathrm{pH} 6$ and $37^{\circ} \mathrm{C}$ $1 \mathrm{~h}$ on $2 \%$ debranched arabinan. (E) Comparative enzymatic activity of xyloglucanases at a concentration of $0.5 \mu \mathrm{M}$ at pH6 and $37^{\circ} \mathrm{C} 1 \mathrm{~h}$ on $2 \%$ xyloglucan. $(\mathrm{F})$ Comparative enzymatic activity of $R$. champanellensis cellulosomal arabinofuranosidases. The enzymes were tested at $\mathrm{pH}$ 6 and $37^{\circ} \mathrm{C}$ for $20 \mathrm{~min}$ with $12.5 \mathrm{mM}$ pNP- $\alpha$-L-arabinofuranoside. Reactions were performed at least twice in triplicate; standard deviations are indicated.

A total of 525 interactions were tested, among them 80 positives (Figs S4 and S5; negative interactions are not shown). Binding affinity partners of 24 dockerins were determined out of the 25 examined.

Glycoside-hydrolases from Group 2 (alignment in Fig. S6) presented various binding profiles (Table 4). Cel5A, Cel9A and Xeg74A dockerins could generally bind all of the cohesins partners (Cohesins A2, B1/B2/B3, B4, B5/B6, $\mathrm{H}$ and I) except for the type I Cohs $C$ and D. The Cel5B dockerin exhibited binding affinity for cohesins B5/B6, $\mathrm{H}$ and I. The Cel9C, Cel48A, Man26B and Xyn10A dockerins were able to bind cohesins A2, B1/B2/ $\mathrm{B} 3, \mathrm{H}$ and I. The Xeg44A dockerin has affinity only to cohesin $\mathrm{H}$. The dockerin of Cel9D exhibited binding affinity for Cohesins B5/B6 only. Finally, the Cel9F and Man5A dockerins interacted with cohesins $A 2, B 1 / B 2 / B 3$ and $\mathrm{H}$.
Glycoside-hydrolases from Groups 3 and 4 were all active except for Cel8A as previously reported (Ben David et al., 2015) (Table 4, Figs S4 and S5). The binding profile of the dockerins matched almost perfectly the abovepredicted interactions. The dockerins of Glc16A (i.e. a cloned portion of the complete enzyme CBM4-Fn3GH9B-Doc-GH16A), Cel9G, Xyn10B-Abf43E, Man26A, Abf43B, GH43C and Arb43D all interacted with CohC, CohD and Cohl. The Arb43A dockerin, which would be predicted to share the same binding profile, interacted strongly only with CohD and weakly with CohC as previously reported (Ben David et al., 2015). However, based on the dockerin sequence, it would seem that it should bind to all three cohesins and not only to cohesin D. Since the interaction with cohesin $D$ is relatively weak, it is therefore likely that the protein may not have been expressed and folded properly. One explanation for the 


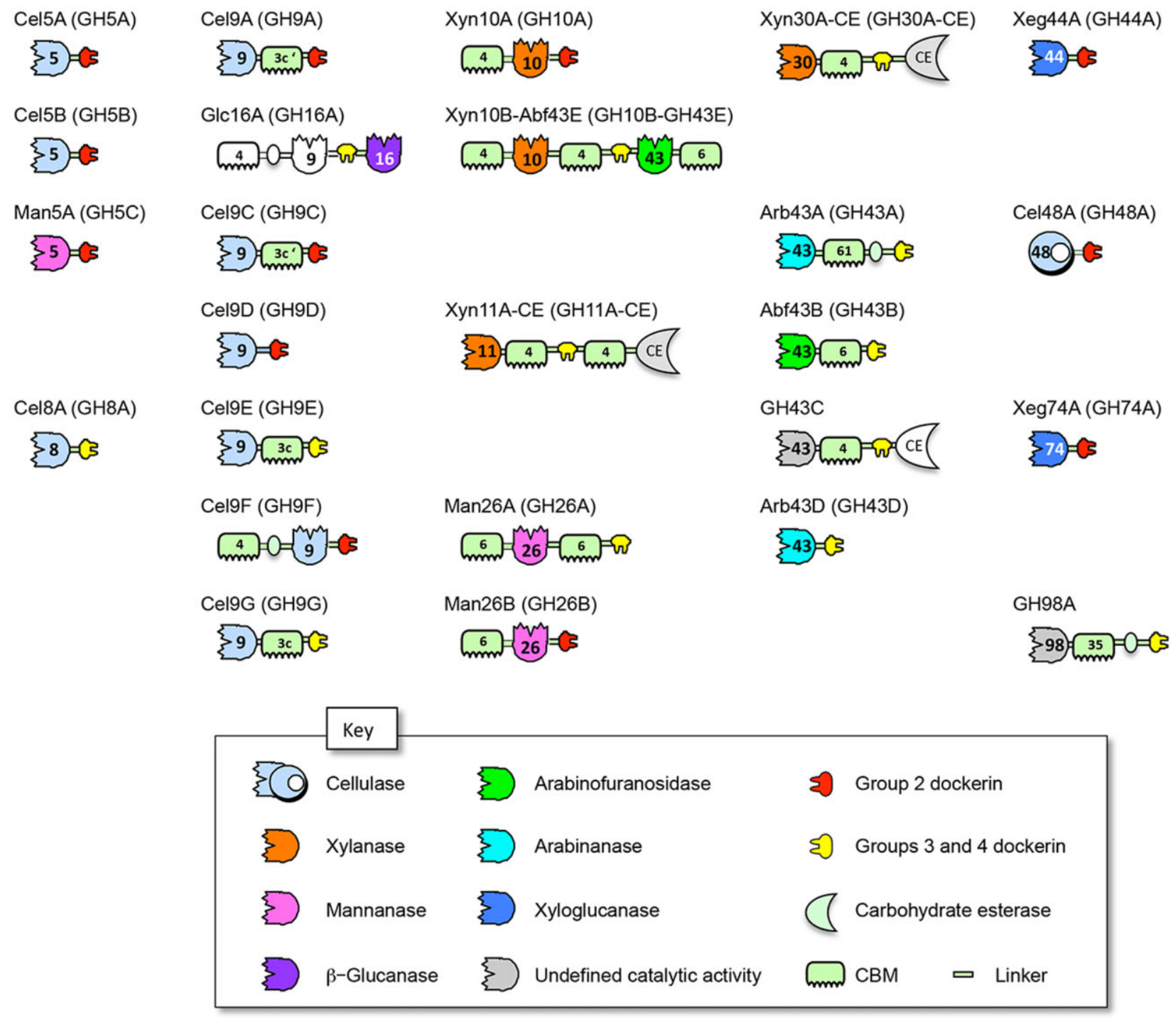

Fig. 3. Schematic representation and proposed nomenclature of the dockerin-containing glycoside hydrolases from $R$. champanellensis. Enzyme activity and dockerin-specificity are colour-coded. GH and CBM families are indicated numerically. Modules shown in white were not expressed in this study.

lack of binding of the Cel8A dockerin and the weak binding of Arb43D dockerin could be the presence of cysteine residues in position 14 that could disturb proper folding in those particular cases.

Xyn11A-CE, Xyn30A-CE and GH98A dockerins interacted selectively with CohC and CohD as predicted from their amino acid sequences.

The Cel9E dockerin, whose dockerin-binding profile remained uncharacterized in the previous work, interacted with CohC and Cohl. The binding to CohC could be attributed to Arg and Lys residues in positions 18 and 19, respectively, and the ability to interact with Cohl could be related to the valine residues in positions 10 and 14 and the aromatic Phe residue, in position 19.

\section{Discussion}

The microbial community that occupies the human gut habitat is known to produce an arsenal of enzymes that together degrade complex carbohydrates from the diet that cannot be hydrolysed by human-based enzymes (Flint et al., 2008), thereby providing supplemental energy sources for the host. Bacteria within this community are believed to have evolved to specialize in certain types of carbohydrate degradation and complement each other (Martens et al., 2011). Bacteroidetes display enzymatic activities for starch, hemicellulose, pectins and glucans (Xu et al., 2003a) but limited ability for cellulose degradation (Robert et al., 2007; McNulty et al., 2013). On the 


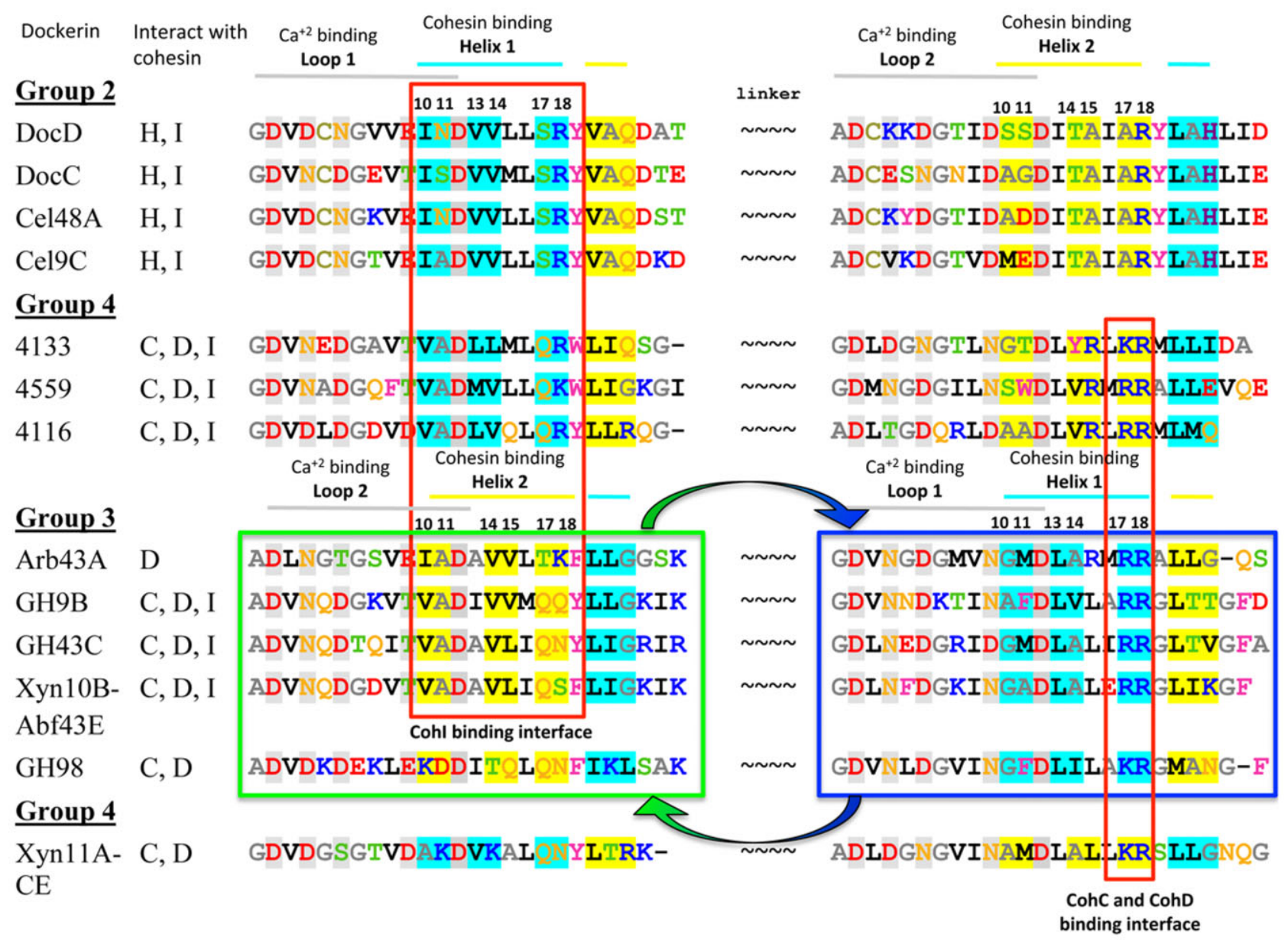

Fig. 4. Proposed twofold alternative specificity mechanism of $R$. champanellensis cohesins C, D and I. Red boxes indicate the residues suspected as responsible for specific cohesin recognition. Residues highlighted in cyan and yellow are involved in the two forms of binding to a cohesin. Note that the two segments of Group 3 dockerins (blue and green boxes, arrows) appear in reversed order, such that their predicted recognition residues align with those of the Group 4 dockerins (yellow). Positions of calcium-binding residues are shown in grey. Numbering indicates the residue positions in the two duplicated segments.

other hand, Firmicutes are able to utilize starch, cellulose and hemicelluloses. They are considered to be more substrate-specific (Salyers et al., 1977; Chassard et al., 2007; 2012; Walker et al., 2011; Ze et al., 2012), and some species among the Firmicutes purportedly represent keystone species in polysaccharide degradation (Ze et al., 2013; Ben David et al., 2015).

The $R$. champanellensis genome contains a repertoire of 12 scaffoldins (Table 3 ), each of which contains various numbers of cohesins from one to seven. In most cases, the scaffoldins also possess a dockerin that will allow interactions with other scaffoldins. The cohesin-dockerin interactions among the various components revealed the possible assembly of a cell-associated cellulosomal complex that could assemble up to 11 enzymes.

In this study, we conducted an extensive, nearcomplete analysis of the cellulosomal enzymatic system of $R$. champanellensis. In addition, the dockerin specifici- ties of 25 enzymes were revealed and were found to be consistent with our overall predictions, based on the sequence similarity between dockerins and recognition residues. The $R$. champanellensis genome contains 65 dockerin-bearing proteins, among which 25 enzymes were characterized in the present study, and 8 scaffoldinborne dockerins were characterized in our previous study (Ben David et al., 2015), in addition to 31 non-glycosidehydrolase dockerin-containing proteins. The dockerin specificities of the latter remain to be elucidated. As in our previous study (Ben David et al., 2015), none of the dockerins examined in this study interacted with five cohesins (namely, CohB6, CohB7, CohF, CohG and CohK). Consequently, their respective binding partner(s) remain as yet unknown.

The set of cellulosomal enzymes in $R$. champanellensis comprises both cellulose- (endoglucanases and exoglucanases) and hemicellulose-degrading activities, 
Table 3. List of the R. champanellensis CBM-fused cohesin proteins used in this article.

\begin{tabular}{lll}
\hline Fused cohesin & Emerging scaffoldin & Modular architecture \\
\hline CBM-CohA2 & ScaA & SIGN X Coh Coh Doc \\
CBM-CohB1/B2/B3 & ScaB & SIGN Coh Coh Coh Coh Coh Coh Coh X Doc \\
CBM-CohB4 & ScaB & SIGN Coh Coh Coh Coh Coh Coh Coh X Doc \\
CBM-CohB5/B6 & ScaB & SIGN Coh Coh Coh Coh Coh Coh Coh X Doc \\
CBM-CohB6 & ScaB & SIGN Coh Coh Coh Coh Coh $\overline{\text { Coh Coh X Doc }}$ \\
CBM-CohB7 & ScaB & SIGN Coh Coh Coh Coh Coh Coh Coh X Doc \\
CBM-CohC & ScaC & SIGN Coh UNK Doc \\
CBM-CohD & ScaD & SIGN Coh Doc \\
CBM-CohE & ScaE & SIGN $\overline{\text { Coh SORT }}$ \\
CohF-CBM & ScaF & SIGN Coh Doc \\
CBM-CohG & ScaG & SIGN Coh Doc \\
CBM-CohH & ScaH & SIGN SGNH Coh Doc \\
CBM-Cohl & Scal & SIGN Coh \\
CohJ1-CBM & ScaJ & SIGN Coh Coh Coh Doc \\
CBM-CohJ2 & ScaJ & SIGN Coh Coh Coh Doc \\
CBM-CohJ3 & ScaJ & SIGN Coh Coh Coh Doc \\
CBM-CohK & ScaK & SIGN Coh GH25 \\
CBM-CohL & ScaL & SIGN UNK NUC Coh Doc \\
CBM-CohCc $(-)$ & Clostridium cellulolyticum CipC & (Negative control)
\end{tabular}

Name and modular architecture of the original scaffoldin are given. Abbreviations: CBM, CBM3a from the C. thermocellum CipA scaffoldin; SIGN, signal peptide; Doc, dockerin; Coh, cohesin; GH, glycoside hydrolase; SGNH, lipases or esterases; SORT, sortase motif; NUC, nucleoporin-like module; UNK, X, unknown.

the latter of which include xylanases, mannanases, arabinanases, xyloglucanases and arabinofuranosidases. One interesting fact is that all members of the cellulase families, i.e. GH8, GH9 and GH48, contain a dockerin module. Moreover, all members of the hemicellulase families, including GH10, GH11, GH30, GH43, GH44 and GH74, are also cellulosomal enzymes. The R. champanellensis genome also contains eight GH5 enzymes, but only three of them are cellulosomal. Sequence alignment and phylogenetic tree analysis of the additional five GH5s with other characterized GH5 enzymes demonstrated that four of them are predicted cellulases and one is consistent with mannanases.

Cellulosome-producing bacteria frequently possess two sets of enzymes, cellulosomal and non-cellulosomal. Clostridium thermocellum, for example, produces two highly active cellulases, Cel48Y and Cel9I, which contain cellulose-specific CBMs instead of dockerins, and are therefore not part of the cellulosome system (Berger et al., 2007). The non-cellulosomal system also includes two GH5s, three GH10s (at least one of which exhibited xylanase activity; Zverlov et al., 2005), one GH43 and several others (Dassa et al., 2012). Intriguingly, R. champanellensis produces cellulosome complexes for its main strategy for both cellulose and hemicellulose degradation, with only a few free enzymes confined to $\mathrm{GH}$ family 5 .

It is interesting to note that representatives of the GH48 and GH9 families were highly upregulated in the proteome of both $R$. champanellensis and $R$. flavefaciens cells (Vodovnik et al., 2013) when grown on cellulose rather than cellobiose. Both of these highly expressed proteins carry dockerins and are thus assumed to be cellulosomal in these two species. Both types of enzymes are typically abundant in cellulosomes, particularly when the parent bacterium is grown on cellulosic substrates (Dror et al., 2003; Berg Miller et al., 2009).

It is also interesting to note that the R. champanellensis genome codes for a GH98 enzyme, which is rare, and this enzyme is also part of the cellulosomal machinery. Thus far, a dockerin-containing GH98 was reported previously only in Clostridium cellulovorans (Cantarel et al., 2009). Ruminococcus albus also produces a GH98, but without a dockerin (Dassa et al., 2014). GH98 enzymes have previously been shown to exhibit blood group endo- $\beta$-galactosidase activity in pathogenic bacteria, although in our particular case the enzyme appeared to be inactive on a colorimetric galactopyranoside-containing substrate.

The enzymes examined in this study exhibit two types of cohesin-dockerin specificities. The specificity type seems to be unrelated to the molecular weight of the proteins but could perhaps be linked to the enzymatic activity, i.e. Group 2 enzymes representing mostly cellulases, and Groups 3 and 4 mostly hemicellulases and non-glycoside hydrolase proteins. These results raise the question why certain enzymes need an adaptor scaffoldin to be integrated into the cellulosomal complex. An interesting observation is that most of the enzymes that bind directly to the scaffoldin (from Group 2) have a particularly long Thr-rich linker that links the dockerin to the catalytic module, which may infer that the adaptor scaffoldins 


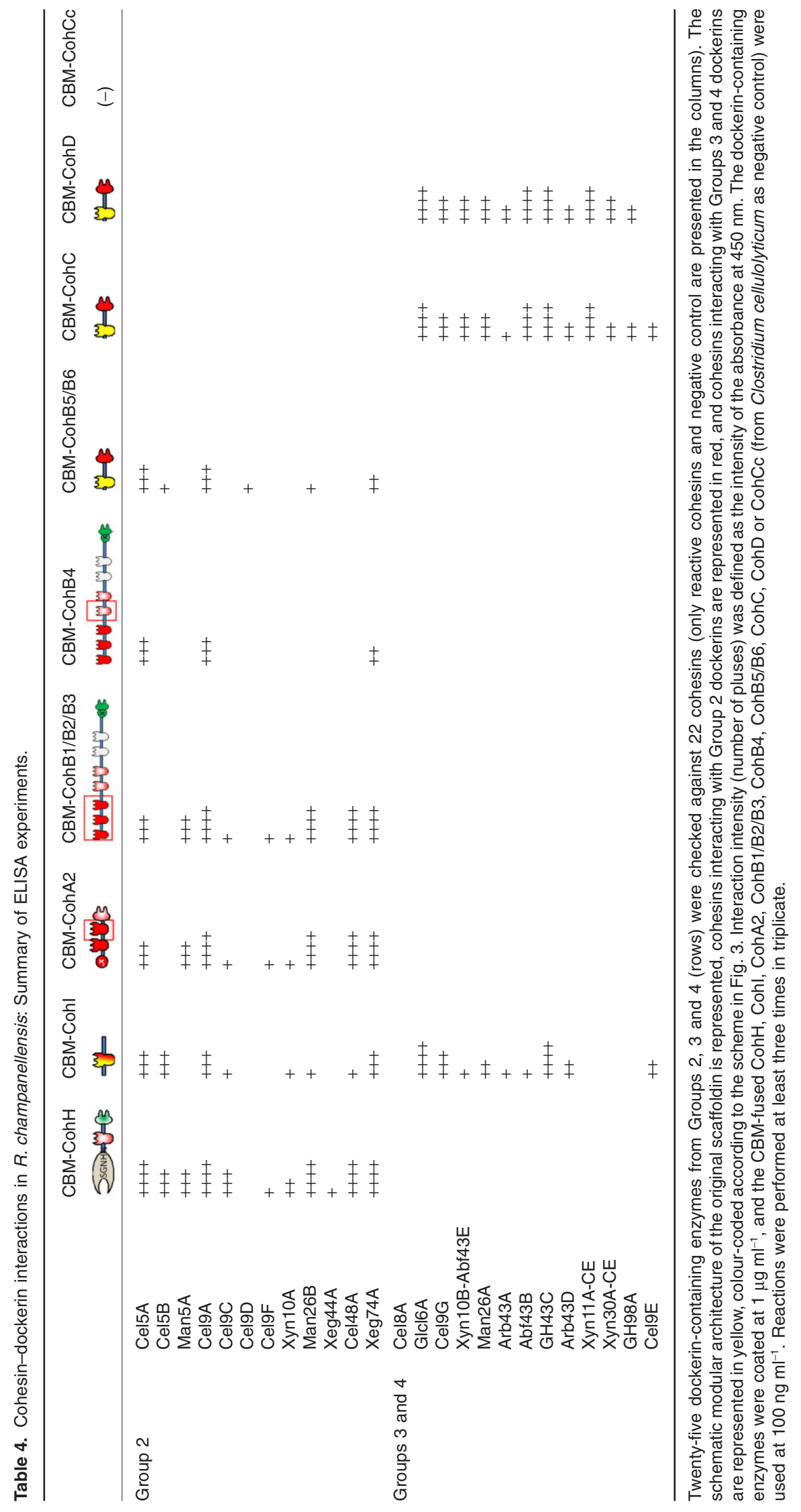


(ScaC and ScaD) also serve as a linker for proteins that lack these types of linker (from Groups 3 and 4).

Similar to the R. champanellensis Sca's C and D, the $R$. flavefaciens $\mathrm{ScaC}$ also serves as an adaptor scaffoldin, which allows many proteins that are not recognized directly by the ScaA cohesins to be bridged into the cellulosome assembly (Rincon et al., 2004). These types of adaptors are different from adaptor scaffoldins that serve to amplify the number of enzymes in the cellulosomal complex ( $\mathrm{Xu}$ et al., 2003b; Dassa et al., 2012). In contrast, monovalent adaptor scaffoldin may be part of a regulatory mechanism for cellulosomal composition.

An interesting fact is that in each of the two specificities, the dockerins did not interact similarly with the various cohesins but presented diverse patterns of affinity. This phenomenon is especially intriguing considering that Group 2 dockerin sequences are very similar. This could reflect an organized manner of integrating enzymes or cellulosomal components in the complex and not a random assembly of the enzymes on the scaffoldin as suggested for cellulosome assembly in other bacteria. Multiple cohesin-dockerin binding specificities have also been demonstrated for different dockerin-carrying enzymes in the phylogenetically related R. flavefaciens (Rincon et al., 2003; Jindou et al., 2006). These results for both Ruminococcus spp. essentially contradict those of a recent study by Hirano and colleagues (Hirano et al., 2015), in which it was suggested that preferential binding of cellulosomal enzymes to the cohesin modules did not result from slight differences in binding affinity but from differences in the length of the inter-cohesin linker: a shorter inter-cohesin linker promoting preferential binding.

Our analyses contribute to a better understanding of the enzymatic degradation of complex carbohydrates by $R$. champanellensis in the human gut. Our findings highlight the importance of the cellulosome paradigm for cellulose and hemicellulose degradation and the controlled assembly of the complex via fine-tuned cohesin-dockerin recognition.

\section{Experimental procedures}

\section{Cloning}

Dockerin-containing glycoside hydrolases were cloned from R. champanellensis genomic DNA using appropriate primers (Table S1) and Phusion High Fidelity DNA polymerase F530-S (New England Biolabs). The genes were restricted using Fastdigest enzymes (Thermo Scientific, USA) and ligated either into pET21a or pET28a using T4 DNA ligase (Fermentas UAB, Vilnius, Lithuania). The constructs were designed to contain a His-tag for subsequent purification.

The CBM-Coh gene cassette (Barak et al., 2005) consists of a family 3 a CBM from the $C$. thermocellum CipA scaffoldin cloned into plasmid pET28a (Novagen, Madison, WI, USA), into which any cohesin gene can be introduced between BamHI and Xhol restriction sites of the plasmid. The CohCBM gene cassette is the same as the CBM-Coh cassette, only in reverse order of the modules. Any cohesin gene can be introduced between $\mathrm{Ncol}$ and $\mathrm{BamHI}$ restriction sites of the plasmid. The full list of fused cohesins used in this article is given in Table 3 .

The PCR products were purified using a HiYield ${ }^{T M}$ Gel/PCR Fragments Extraction Kit (Real Biotech Corporation, RBC, Taiwan), and plasmids were extracted using Qiagen Miniprep Kit (Netherlands). The cloning of each gene was confirmed by DNA sequencing. Competent E. coli XL1 competent cells were used for plasmid transformation.

\section{Recombinant protein expression and purification}

The E. coli BL21 (DE3) cells were transformed with the desired plasmid and plated onto LB-kanamycin plates. The cells producing GH5B-, GH8A-, GH9A-, GH9C-, GH9D-, GH9E-, GH9F-, GH9G-, GH10A-, GH10B/GH43E-, GH11A/ CE-, GH16-, GH43C- and GH74A-containing enzymes and ScaL were grown in $50 \mathrm{ml} \mathrm{LB}$ (Luria Broth) and $2 \mathrm{mM} \mathrm{CaCl}_{2}$ (to facilitate proper folding of the dockerin) at $37^{\circ} \mathrm{C}$ until $\mathrm{A}_{600} \approx 0.8-1$ and induced by adding $0.1 \mathrm{mM}$ (final concentration) isopropyl-1-thio- $\beta$-D-galactoside (IPTG) (Fermentas UAB). Cell growth was continued at $16^{\circ} \mathrm{C}$ overnight. Cells producing GH5A, GH5C, GH26A, GH26B, GH30A-CE, GH43A, GH43B, GH43D, GH44A, GH48A or GH98A were grown in $50 \mathrm{ml}$ tryptone yeast glucose medium supplemented with $2 \mathrm{mM} \mathrm{CaCl}$ at $37^{\circ} \mathrm{C}$ until $\mathrm{A}_{600} \approx 0.8-1$ and induced by adding $0.1 \mathrm{mM}$ IPTG. Growth was continued $3 \mathrm{~h}$ at $37^{\circ} \mathrm{C}$. Cells were harvested by centrifugation at 5000 r.p.m. for 5 min. Pelleted cells were re-suspended in $1 \mathrm{ml}$ TBS (Tris-buffered saline, $137 \mathrm{mM} \mathrm{NaCl}, 2.7 \mathrm{mM} \mathrm{KCL}, 25 \mathrm{mM}$ Tris- $\mathrm{HCl}$, $\mathrm{pH}=7.4$ ).

The His-tagged proteins were either purified on a nickelnitrilotriacetic acid (Ni-NTA) column (Qiagen), as reported earlier (Caspi et al., 2006) or small-scale purified using Ni-NTA spin columns (Qiagen). The cohesin-containing protein supernatant fluids were added to $2 \mathrm{~g}$ of macroporous bead cellulose pre-swollen gel (IONTOSORB, Usti nad Labem, Czech Republic), and incubated for $1 \mathrm{~h}$, with rotation at $4^{\circ} \mathrm{C}$. The mixture was then loaded onto a gravity column, and washed with $100 \mathrm{ml}$ of TBS containing $1 \mathrm{M} \mathrm{NaCl}$, and then washed with $100 \mathrm{ml}$ TBS. Three $5 \mathrm{ml}$ elutions of $1 \%$ triethanolamine were then collected. The fractions were subjected to SDS-PAGE in order to assess protein purity, and then dialysed with TBS.

Purity of the recombinant proteins was tested by SDSPAGE on 10\% acrylamide gels. Protein concentration was estimated by absorbance $(280 \mathrm{~nm})$ based on the known amino acid composition of the protein using the PROTPARAM tool (http://www.expasy.org/tools/protparam.html). Proteins were stored in $50 \%(\mathrm{v} / \mathrm{v})$ glycerol at $-20^{\circ} \mathrm{C}$.

\section{Enzymatic activity assay}

All assays were performed at least twice in triplicate. The different proteins were tested against several potential substrates according to the GH family (Cantarel et al., 2009) and 
at a $\mathrm{pH}$ corresponding to the optimal $\mathrm{pH}$ generally observed for these enzymatic activities in previous studies. All enzymes were tested at a concentration of $0.5 \mu \mathrm{M}$ at $37^{\circ} \mathrm{C}$. Cellulases were tested at $\mathrm{pH} 5$ (buffer acetate $50 \mathrm{mM}$ final concentration), for either $1 \mathrm{~h}$ with $2 \%$ CMC (VWR International, England) or in 10\% Avicel for $24 \mathrm{~h}$ (FMC, Delaware USA). Xylanases were tested at $\mathrm{pH} 6$ (buffer citrate $50 \mathrm{mM}$ final concentration) for $1 \mathrm{~h}$ with $2 \%$ beechwood xylan (Sigma). $\beta$-glucanase were tested on $\beta$-D glucan from barley (Sigma) for $1 \mathrm{~h}$ at $\mathrm{pH} 5$ (buffer acetate $50 \mathrm{mM}$ final concentration). Arabinanases were tested at $\mathrm{pH} 6$ (buffer citrate $50 \mathrm{mM}$ final concentration), for $1 \mathrm{~h}$ with $2 \%$ debranched arabinan (Megazyme, Ireland). Mannanases were tested at $\mathrm{pH} 5$ (buffer acetate $50 \mathrm{mM}$ final concentration) for $1 \mathrm{~h}$ with $1 \%$ locus bean gum. The xyloglucanase was examined with $2 \%$ xyloglucan (Megazyme) for $1 \mathrm{~h}$ at pH 6 (buffer citrate $50 \mathrm{mM}$ final concentration). Enzymatic reactions were terminated by transferring the tubes to an ice-water bath, and the tubes were centrifuged for 2 min at 14000 r.p.m. at room temperature. Enzymatic activity was then determined quantitatively by measuring the soluble reducing sugars released from the polysaccharide substrates by the dinitrosalicylic acid (DNS) method (Miller, 1959; Ghose, 1987). The DNS solution $(150 \mu \mathrm{l})$ was added to $100 \mu \mathrm{l}$ of sample, and after boiling the reaction mixture for $10 \mathrm{~min}$, absorbance at $540 \mathrm{~nm}$ was measured. Sugar concentrations were determined using a glucose standard curve. The colorimetric substrate, $p N P-\alpha-$ L-arabinofuranoside (pNPA) (Sigma), was used at $12.5 \mathrm{mM}$ and $\mathrm{pH} 6$ (50 $\mathrm{mM}$ citrate buffer) in a reaction mixture containing $0.5 \mu \mathrm{M}$ enzyme, and the tubes were incubated for $20 \mathrm{~min}$ at $37^{\circ} \mathrm{C}$.

Chitin, laminarin, pNP- $\beta$-D-glucopyranoside and pNP- $\beta$-Dcellobioside (Sigma) were also used for substrate specificity determination.

\section{Affinity-based ELISA}

The matching fusion-protein procedure of Barak and colleagues (Barak et al., 2005; Caspi et al., 2006) was followed to determine cohesin-dockerin specificity of interaction. Dockerin-containing enzymes were immobilized on the plate at a concentration of $1 \mu \mathrm{g} \mathrm{ml}^{-1}\left(100 \mu \mathrm{l} \mathrm{well}^{-1}\right)$ in $0.1 \mathrm{M}$ sodium carbonate $(\mathrm{pH} 9)$ and incubated at $4^{\circ} \mathrm{C}$ overnight. The following steps were performed at room temperature for $1 \mathrm{~h}$ with all reagents at a volume of $100 \mu \mathrm{lwell}^{-1}$, with a three-times repeated washing step $\left(300 \mu \mathrm{l} \mathrm{well}^{-1}\right.$ blocking buffer without BSA) included after each step. The coating solution was discarded, and blocking buffer (TBS, $10 \mathrm{mM} \mathrm{CaCl}, 0.05 \%$ Tween 20, $\%$ BSA) was added. The blocking buffer was discarded, and the desired CBMCoh(s), diluted to concentrations of $100 \mathrm{ng} \mathrm{m}^{-1}$ in blocking buffer, were added. Rabbit anti-CBM antibody (diluted $1: 3000)$ was used as the primary antibody preparation, and the secondary antibody preparation was horseradish peroxidase (HRP)-labelled anti-rabbit antibody diluted 1:10 000 in blocking buffer. Substrate-Chromogen TMB (Dako, Agilent Technologies, USA) was added at $100 \mu \mathrm{lwell}{ }^{-1}$, and the reaction was carried out for $2 \mathrm{~min}$ before colour formation was terminated upon addition of $1 \mathrm{M} \mathrm{H}_{2} \mathrm{SO}_{4}\left(50 \mu \mathrm{l}\right.$ well $\left.{ }^{-1}\right)$, and the absorbance was measured at $450 \mathrm{~nm}$ using a tunable microplate reader.
Proteomic analysis of $\mathrm{R}$. champanellensis $18 \mathrm{P} 13$

The R. champanellensis 18P13 cultures were grown anaerobically $\left(37^{\circ} \mathrm{C}\right)$ in $800 \mathrm{ml}$ of basal yeast extract-casein hydrolysate-fatty acids (YCFA) medium (Lopez-Siles et al., 2012) containing $1 \%$ clarified rumen fluid with either $0.5 \%$ cellobiose or $0.5 \%$ of filter paper cellulose cut into $1 \mathrm{~cm}$ squares (Whatman No.1) for $48 \mathrm{~h}$ and $96 \mathrm{~h}$ respectively. Samples were analysed from duplicate biological repeats, with three technical replicates for each gel separation, such that comparison was made between six gel separations from each growth condition. The cellulose-grown cells were harvested following vigorous shaking and allowing the substrate to sediment for a period of $10 \mathrm{~min}$. The cells from both the cellobiose- and cellulose-grown cultures were harvested as described by Vodovnik and colleagues (2013). Equivalent levels of proteins in Rabilloud buffer were separated by twodimensional gel electrophoresis, and gels were imaged as described previously (Vodovnik et al., 2013). The gels were analysed with PD Quest software (Bio-Rad). Spots of interest were excised from the gels manually, then processed and identified by Nano LC MS/MS as described previously (Vodovnik et al., 2013).

\section{Acknowledgements}

This research was supported by the United States-Israel Binational Science Foundation (BSF), Jerusalem, Israel, and by a grant (No. 1349) to EAB from the Israel Science Foundation (ISF). Additional support was obtained from the establishment of an Israeli Center of Research Excellence (I-CORE Center No. 152/11) managed by the Israel Science Foundation. The authors also appreciate the support of the European Union, Area NMP.2013.1.1-2: Self-assembly of naturally occurring nanosystems: CellulosomePlus Project Number: 604530 and an ERA-IB Consortium (EIB.12.022), acronym FiberFuel. In addition, EAB is grateful for a grant from the F. Warren Hellman Grant for Alternative Energy Research in Israel in support of alternative energy research in Israel administered by the Israel Strategic Alternative Energy Foundation (I-SAEF). HJF acknowledges support from BBSRC Grant No BB/L009951/1, from the Scottish Government Food, Land and People program, and from the Society for Applied Microbiology. Thanks are due to Fergus Nicol and Louise Cantlay for proteomic analysis. EAB is the incumbent of The Maynard I. and Elaine Wishner Chair of Bio-organic Chemistry.

\section{References}

Barak, Y., Handelsman, T., Nakar, D., Mechaly, A., Lamed, R., Shoham, Y., and Bayer, E.A. (2005) Matching fusion protein systems for affinity analysis of two interacting families of proteins: the cohesin-dockerin interaction. $\mathrm{J} \mathrm{Mol}$ Recognit 18: 491-501.

Bayer, E.A., Belaich, J.-P., Shoham, Y., and Lamed, R. (2004) The cellulosomes: multi-enzyme machines for degradation of plant cell wall polysaccharides. Annu Rev Microbiol 58: 521-554. 
Ben David, Y., Dassa, B., Borovok, I., Lamed, R., Koropatkin, N.M., Martens, E.C., et al. (2015) Ruminococcal cellulosome systems from rumen to human. Environ Microbiol 17: 3407-3426.

Berg Miller, M.E., Antonopoulos, D.A., Rincon, M.T., Band, M., Bari, A., Akraiko, T., et al. (2009) Diversity and strain specificity of plant cell wall degrading enzymes revealed by the draft genome of Ruminococcus flavefaciens FD-1. PLOS ONE 4: e6650.

Berger, E., Zhang, D., Zverlov, V.V., and Schwarz, W.H. (2007) Two noncellulosomal cellulases of Clostridium thermocellum, Cel9I and Cel48Y, hydrolyse crystalline cellulose synergistically. FEMS Microbiol Lett 268: 194201.

Bras, J.L., Alves, V.D., Carvalho, A.L., Najmudin, S., Prates, J.A., Ferreira, L.M., et al. (2012) Novel Clostridium thermocellum type I cohesin-dockerin complexes reveal a single binding mode. J Biol Chem 287: 4439444405.

Cantarel, B.L., Coutinho, P.M., Rancurel, C., Bernard, T., Lombard, V., and Henrissat, B. (2009) The carbohydrateactive enzymes database (CAZy): an expert resource for glycogenomics. Nucleic Acids Res 37: D233-D238.

Carvalho, A.L., Dias, F.M., Prates, J.A., Nagy, T., Gilbert, H.J., Davies, G.J., et al. (2003) Cellulosome assembly revealed by the crystal structure of the cohesin-dockerin complex. Proc Natl Acad Sci USA 100: 13809-13814.

Carvalho, A.L., Dias, F.M., Nagy, T., Prates, J.A., Proctor, M.R., Smith, N., et al. (2007) Evidence for a dual binding mode of dockerin modules to cohesins. Proc Natl Acad Sci USA 104: 3089-3094.

Caspi, J., Irwin, D., Lamed, R., Shoham, Y., Fierobe, H.-P., Wilson, D.B., and Bayer, E.A. (2006) Thermobifida fusca family- 6 cellulases as potential designer cellulosome components. Biocatal Biotransformation 24: 3-12.

Chassard, C., Goumy, V., Leclerc, M., Del'homme, C., and Bernalier-Donadille, A. (2007) Characterization of the xylan-degrading microbial community from human faeces. FEMS Microbiol Ecol 61: 121-131.

Chassard, C., Delmas, E., Robert, C., Lawson, P.A., and Bernalier-Donadille, A. (2012) Ruminococcus champanellensis sp. nov., a cellulose-degrading bacterium from human gut microbiota. Int J Syst Evol Microbiol 62: 138-143.

Dassa, B., Borovok, I., Lamed, R., Henrissat, B., Coutinho, P., Hemme, C.L., et al. (2012) Genome-wide analysis of Acetivibrio cellulolyticus provides a blueprint of an elaborate cellulosome system. BMC Genomics 13: 1-13.

Dassa, B., Borovok, I., Ruimy-Israeli, V., Lamed, R., Flint, H.J., Duncan, S.H., et al. (2014) Rumen cellulosomics: divergent fiber-degrading strategies revealed by comparative genome-wide analysis of six ruminococcal strains. PLOS ONE 9: e99221.

Ding, S.Y., Rincon, M.T., Lamed, R., Martin, J.C., McCrae, S.I., Aurilia, V., et al. (2001) Cellulosomal scaffoldin-like proteins from Ruminococcus flavefaciens. J Bacteriol 183: 1945-1953.

Dror, T.W., Morag, E., Rolider, A., Bayer, E.A., Lamed, R., and Shoham, Y. (2003) Regulation of the cellulosomal CelS (cel48A) gene of Clostridium thermocellum is growth rate dependent. J Bacteriol 185: 3042-3048.
Flint, H.J., Bayer, E.A., Rincon, M.T., Lamed, R., and White, B.A. (2008) Polysaccharide utilization by gut bacteria: potential for new insights from genomic analysis. Nat Rev Microbiol 6: 121-131.

Ghose, T.K. (1987) Measurements of cellulase activity. Pure Appl Chem 59: 257-268.

Goodman, A.L., McNulty, N.P., Zhao, Y., Leip, D., Mitra, R.D., Lozupone, C.A., et al. (2009) Identifying genetic determinants needed to establish a human gut symbiont in its habitat. Cell Host Microbe 6: 279-289.

Hirano, K., Nihei, S., Hasegawa, H., Haruki, M., and Hirano, N. (2015) Stoichiometric assembly of cellulosome generates maximum synergy for the degradation of crystalline cellulose, as revealed by in vitro reconstitution of the Clostridium thermocellum cellulosome. Appl Environ Microbiol 81: 4756-4766.

Jindou, S., Borovok, I., Rincon, M.T., Flint, H.J., Antonopoulos, D.A., Berg, M.E., et al. (2006) Conservation and divergence in cellulosome architecture between two strains of Ruminococcus flavefaciens. J Bacteriol 188: 7971-7976.

Kerckhoffs, A.P., Ben-Amor, K., Samsom, M., van der Rest, M.E., de Vogel, J., Knol, J., and Akkermans, L.M. (2011) Molecular analysis of faecal and duodenal samples reveals significantly higher prevalence and numbers of Pseudomonas aeruginosa in irritable bowel syndrome. J Med Microbiol 60: 236-245.

Lamed, R., Setter, E., and Bayer, E.A. (1983) Characterization of a cellulose-binding, cellulase-containing complex in Clostridium thermocellum. J Bacteriol 156: 828-836.

Lee, Y.K., and Mazmanian, S.K. (2010) Has the microbiota played a critical role in the evolution of the adaptive immune system? Science 330: 1768-1773.

Lopez-Siles, M., Khan, T.M., Duncan, S.H., Harmsen, H.J., Garcia-Gil, L.J., and Flint, H.J. (2012) Cultured representatives of two major phylogroups of human colonic Faecalibacterium prausnitzii can utilize pectin, uronic acids, and host-derived substrates for growth. Appl Environ Microbiol 78: 420-428.

Lytle, B., Myers, C., Kruus, K., and Wu, J.H.D. (1996) Interactions of the CelS binding ligand with various receptor domains of the Clostridium thermocellum cellulosomal scaffolding protein, CipA. J Bacteriol 178: 12001203.

McNulty, N.P., Wu, M., Erickson, A.R., Pan, C., Erickson, B.K., Martens, E.C., et al. (2013) Effects of diet on resource utilization by a model human gut microbiota containing Bacteroides cellulosilyticus WH2, a symbiont with an extensive glycobiome. PLOS Biol 11: e1001637.

Martens, E.C., Lowe, E.C., Chiang, H., Pudlo, N.A., Wu, M., McNulty, N.P., et al. (2011) Recognition and degradation of plant cell wall polysaccharides by two human gut symbionts. PLoS Biol 9: e1001221.

Mechaly, A., Yaron, S., Lamed, R., Fierobe, H.P., Belaich, A., Belaich, J.P., et al. (2000) Cohesin-dockerin recognition in cellulosome assembly: experiment versus hypothesis. Proteins 39: 170-177.

Mechaly, A., Fierobe, H.-P., Belaich, A., Belaich, J.-P., Lamed, R., Shoham, Y., and Bayer, E.A. (2001) Cohesindockerin interaction in cellulosome assembly: a single hydroxyl group of a dockerin domain distinguishes 
between non-recognition and high-affinity recognition (Erratum). J Biol Chem 276: 19678.

Miller, G.L. (1959) Use of dinitrosalicylic acid reagent for determination of reducing sugar. Anal Biochem 31: 426428.

Morais, S., Barak, Y., Lamed, R., Wilson, D.B., Xu, Q., Himmel, M.E., and Bayer, E.A. (2012) Paradigmatic status of an endo- and exoglucanase and its effect on crystalline cellulose degradation. Biotechnol Biofuels 5: 1-9.

Pages, S., Belaich, A., Belaich, J.-P., Morag, E., Lamed, R., Shoham, Y., and Bayer, E.A. (1997) Species-specificity of the cohesin-dockerin interaction between Clostridium thermocellum and Clostridium cellulolyticum: prediction of specificity determinants of the dockerin domain. Proteins 29: 517-527.

Pinheiro, B.A., Gilbert, H.J., Sakka, K., Sakka, K., Fernandes, V.O., Prates, J.A., et al. (2009) Functional insights into the role of novel type I cohesin and dockerin domains from Clostridium thermocellum. Biochem J 424: 375-384.

Rincon, M.T., Ding, S.Y., McCrae, S.I., Martin, J.C., Aurilia, V., Lamed, R., et al. (2003) Novel organization and divergent dockerin specificities in the cellulosome system of Ruminococcus flavefaciens. J Bacteriol 185: 703713.

Rincon, M.T., Martin, J.C., Aurilia, V., McCrae, S.I., Rucklidge, G.J., Reid, M.D., et al. (2004) ScaC, an adaptor protein carrying a novel cohesin that expands the dockerinbinding repertoire of the Ruminococcus flavefaciens 17 cellulosome. J Bacteriol 186: 2576-2585.

Rincon, M.T., Dassa, B., Flint, H.J., Travis, A.J., Jindou, S., Borovok, I., et al. (2010) Abundance and diversity of dockerin-containing proteins in the fiber-degrading rumen bacterium, Ruminococcus flavefaciens FD-1. PLOS ONE 5: e12476.

Robert, C., Chassard, C., Lawson, P.A., and Bernalier-Donadille, A. (2007) Bacteroides cellulosilyticus sp. nov., a cellulolytic bacterium from the human gut microbial community. Int J Syst Evol Microbiol 57: 15161520.

Salyers, A.A., West, S.E., Vercellotti, J.R., and Wilkins, T.D. (1977) Fermentation of mucins and plant polysaccharides by anaerobic bacteria from the human colon. Appl Environ Microbiol 34: 529-533.

Turnbaugh, P.J., Ley, R.E., Mahowald, M.A., Magrini, V., Mardis, E.R., and Gordon, J.I. (2006) An obesityassociated gut microbiome with increased capacity for energy harvest. Nature 444: 1027-1031.

Vaarala, O. (2012) Gut microbiota and type 1 diabetes. Rev Diabet Stud 9: 251-259.

Vazana, Y., Morais, S., Barak, Y., Lamed, R., and Bayer, E.A. (2010) Interplay between Clostridium thermocellum family 48 and family 9 cellulases in cellulosomal versus noncellulosomal states. Appl Environ Microbiol 76: 32363243.

Vodovnik, M., Duncan, S.H., Reid, M.D., Cantlay, L., Turner, K., Parkhill, J., et al. (2013) Expression of cellulosome components and type IV pili within the extracellular proteome of Ruminococcus flavefaciens 007. PLoS ONE 8: e65333.

Voronov-Goldman, M., Yaniv, O., Gul, O., Yoffe, H., Salama-Alber, O., Slutzki, M., et al. (2015) Standalone cohesin as a molecular shuttle in cellulosome assembly. FEBS Lett 589: 1569-1576.

Walker, A.W., Ince, J., Duncan, S.H., Webster, L.M., Holtrop, G., Ze, X., et al. (2011) Dominant and diet-responsive groups of bacteria within the human colonic microbiota. ISME J 5: 220-230.

Xu, J., Bjursell, M.K., Himrod, J., Deng, S., Carmichael, L.K., Chiang, H.C., et al. (2003a) A genomic view of the humanBacteroides thetaiotaomicron symbiosis. Science 299: 2074-2076.

Xu, Q., Gao, W., Ding, S.Y., Kenig, R., Shoham, Y., Bayer, E.A., and Lamed, R. (2003b) The cellulosome system of Acetivibrio cellulolyticus includes a novel type of adaptor protein and a cell surface anchoring protein. $J$ Bacteriol 185: 4548-4557.

Yaron, S., Morag, E., Bayer, E.A., Lamed, R., and Shoham, Y. (1995) Expression, purification and subunit-binding properties of cohesins 2 and 3 of the Clostridium thermocellum cellulosome. FEBS Lett 360: 121-124.

Young, G.P., Hu, Y., Le Leu, R.K., and Nyskohus, L. (2005) Dietary fibre and colorectal cancer: a model for environment-gene interactions. Mol Nutr Food Res 49: 571-584.

Ze, X., Duncan, S.H., Louis, P., and Flint, H.J. (2012) Ruminococcus bromii is a keystone species for the degradation of resistant starch in the human colon. ISME J 6 : 1535-1543.

Ze, X., Le Mougen, F., Duncan, S.H., Louis, P., and Flint, H.J. (2013) Some are more equal than others: the role of 'keystone' species in the degradation of recalcitrant substrates. Gut Microbes 4: 236-240.

Zhang, X.Z., Sathitsuksanoh, N., and Zhang, Y.H. (2010) Glycoside hydrolase family 9 processive endoglucanase from Clostridium phytofermentans: heterologous expression, characterization, and synergy with family 48 cellobiohydrolase. Bioresour Technol 101: 5534-5538.

Zverlov, V.V., Schantz, N., Schmitt-Kopplin, P., and Schwarz, W.H. (2005) Two new major subunits in the cellulosome of Clostridium thermocellum: Xyloglucanase Xgh74A and endoxylanase Xyn10D. Microbiology 151: 33953401.

\section{Supporting information}

Additional Supporting Information may be found in the online version of this article at the publisher's web-site:

Fig. S1. Purity of the recombinant enzymes after Ni-NTA purification as assessed by SDS-PAGE gels (10\% acrylamide).

Fig. S2. Comparative proteome of (A) cellobiose and (B) filter paper cellulose-grown Ruminococcus champanellensis 18P13. Spot F1 = Cel9F and Spot F2 = Cel48A.

Fig. S3. New division of $R$. champanellensis Groups 3 and 4 dockerins. The dockerins of Groups 3 and 4 were re-divided based on the finding of the alternative-binding mode (Fig. 4). Positions of the putative cohesin recognition residues are highlighted in cyan for the first helix and in yellow for the second helix. Proteins highlighted in green were examined in our previous study (Ben David et al., 


\section{S. Moraïs et al.}

2015), and proteins highlighted in blue were topics of the present study.

Fig. S4. Affinity-based ELISA with Group 2 enzymes. The dockerin-containing enzymes were coated at $1 \mu \mathrm{g} \mathrm{ml}^{-1}$, and the CBMs fused to CohH, Cohl, CohA2, CohB1/B2/B3, CohB4, CohB5/B6 or CohCc (from Clostridium cellulolyticum as negative control) were used at $100 \mathrm{ng} \mathrm{ml}^{-1}$. Reactions were performed at least three times in triplicate; standard deviations are indicated.

Fig. S5. Affinity-based ELISA with Groups 3 and 4 enzymes. The dockerin-containing enzymes were coated at $1 \mu \mathrm{g} \mathrm{ml}^{-1}$, and the CBMs fused to CohC, CohD, CohH or CohCc (from C. cellulolyticum as negative control) were used at $100 \mathrm{ng} \mathrm{ml}^{-1}$. Reactions were performed at least three times in triplicate; standard deviations are indicated.
Fig. S6. R. champanellensis dockerin Group 2 alignment. The 17 dockerin sequences of $R$. champanellensis were aligned, using bioinformatics-based criteria. Dockerins selected for this study are highlighted in blue and those highlighted in green were also assayed in our previous study (Ben David et al., 2015) (see Table 1 for Gl number of the parent proteins). Positions of calcium binding residues are shown in cyan, and putative recognition residues are shown in yellow. Protein names highlighted green were examined in our previous study (Ben David et al., 2015), and protein names highlighted in blue were topics of the present study. Table S1. Primers used in the study (restrictions sites represented in upper cases). 